\title{
Uncertainty Margins for Probabilistic AC Security Assessment
}

\section{Conference Paper}

\section{Author(s):}

Qu, Haoyuan; Roald, Line; Andersson, Göran

Publication date:

2015

Permanent link:

https://doi.org/10.3929/ethz-a-010607943

Rights / license:

In Copyright - Non-Commercial Use Permitted

Originally published in:

https://doi.org/10.1109/PTC.2015.7232297 


\title{
Uncertainty Margins for Probabilistic AC Security Assessment
}

\author{
Haoyuan Qu, Line Roald, Student Member, IEEE, Göran Andersson, Fellow, IEEE \\ Power Systems Laboratory, Department of Electrical Engineering, ETH Zurich, Switzerland \\ Email: haqu@ student.ethz.ch, \{roald | andersson\}@eeh.ee.ethz.ch
}

\begin{abstract}
In the past few years, the share of renewable power generation has been growing significantly, leading to increased uncertainties in power system operation. In this paper, the effect of wind in-feed uncertainties on power flow in the $\mathrm{AC}$ grid is investigated. The wind power in-feed deviations from initial forecast are modelled as Gaussian random variables. To assess the influence of wind power deviations on power flows, sensitivity factors based on a linearised version of the $\mathrm{AC}$ power flow equations are used. These factors are then applied in the calculation of appropriate security margins needed for keeping the system secure in the presence of wind power fluctuations. The modelling methods are implemented on the IEEE RTS96 test system with additional wind power in-feed. Simulation results show that the proposed method based on linearised AC power flow can estimate uncertainty margins for active power more accurately than the DC approximation model, and also provides satisfactory estimation of uncertainty margins for apparent power.
\end{abstract}

\section{INTRODUCTION}

Public concerns for the environment and climate change have largely stimulated the deployment of Renewable Energy Sources (RES) for electricity generation. In the past decade, global installed wind power capacity has increased rapidly. Wind energy has also become one of the major sources of electrical energy in countries such as Denmark and Spain [1]. Large penetration of wind power poses new challenges to the secure operation of electric power systems. When the wind power in-feeds deviate from the forecast values, the deviations lead to a change in the overall generation dispatch. The resulting variations in the line flows might put system security at risk, since unexpected line flow increases due to RES fluctuations may result in violations of the line capacity limits. Therefore, it is important to investigate the effect of fluctuating wind power in-feed on the line flows and to define appropriate security margins.

Optimal Power Flow (OPF) is used to obtain an optimal solution to the generation dispatch problem, subject to transmission constraints. To account for wind in-feed uncertainty within the OPF, a chance-constrained OPF is introduced in [2]-[6]. The chance constraints limit the probability of constraint violation, and thus provide probabilistic guarantees for the feasibility of the solution. In [5], DC sensitivity factors are used to estimate the influence of wind in-feed deviations on line flows for the formulation of a probabilistic DC OPF. It was observed that accounting for wind power uncertainty leads to a decrease in available transmission capacity, i.e., to the introduction of a security margin against uncertain in-feeds. This type of margin is called an uncertainty margin.

The objective of this paper is to define similar uncertainty margins for use in $\mathrm{AC}$ power flow. The uncertainty margins are calculated based on AC sensitivity factors called AC Power Transfer Distribution Factors (AC-PTDFs), which are derived using a linearisation of the $\mathrm{AC}$ power flow equations. The use of AC-PTDFs to assess the changes in line flows was proposed in [7], where the factors were applied in the evaluation of the influence of power transactions in a liberalized market. It was found that the AC-PTDFs have a higher accuracy than DCPTDFs. Here, the AC-PTDFs are used to investigate the effect of random variations in wind power in-feed on the line flows. The accuracy of the resulting uncertainty margins compared with the full AC power flow equations and the DC power flow approximation is investigated.

The contributions of this paper are threefold: First, AC-PTDFs are used in the context of wind power deviations to obtain a linear relationship between the wind power deviations and the line flow changes, both in active and apparent power flow (Section II). Second, this linear relationship is used to define AC uncertainty margins that can be incorporated within an AC OPF framework (Section III). Finally, the accuracy of the method is assessed through a comparison with the full, nonlinear AC power flow and the original DC approach (Section IV). Section V summarizes and concludes.

\section{Modelling the Influence of Wind Power IN-FEEDS ON LINE FLOWS}

In this section, the modelling method of the wind in-feed uncertainty as well as its impact on line flows are presented. To describe the method, we consider a system consisting of $N_{B}$ buses and $N_{\ell}$ branches. There are $N_{G}$ generators, $N_{W}$ wind power plants and $N_{L}$ loads connected to the system.

\section{A. Wind Power Modelling}

In order to incorporate wind in-feed into day-ahead planning, wind power is usually forecasted using different statistical methods. However, actual wind productions $\mathbf{P}_{W} \in \mathbb{R}^{N_{W}}$ deviate from the forecast values $\mathbf{P}_{\mathrm{W}}^{f c} \in \mathbb{R}^{\mathrm{N}_{\mathrm{W}}}$. This deviation is denoted as forecast errors $\Delta \mathbf{P}_{\mathrm{W}} \in \mathbb{R}^{\mathrm{N}_{\mathrm{W}}}$. Actual wind power in-feeds can be expressed as the sum of forecast values and forecast errors:

$$
\mathbf{P}_{\mathrm{W}}=\mathbf{P}_{\mathrm{W}}^{f c}+\Delta \mathbf{P}_{\mathrm{W}} \text {. }
$$


In this paper, the forecast errors $\Delta \mathbf{P}_{\mathrm{W}}$ are represented as zeromean Gaussian random variables with the covariance matrix $\Sigma_{\mathrm{W}} \in \mathbb{R}^{N_{\mathrm{W}} \times \mathrm{N}_{\mathrm{W}}}$ indicating the uncertainty level of forecast.

\section{B. Compensation of Power Imbalances}

Deviations from the wind power forecast are compensated by conventional generators in the system. If the actual wind power output is larger than the forecast value, other generators will reduce their production proportionally in order to maintain an overall power balance, and vice versa. Here, Generation Shift Factors (GSFs) [8] are introduced to describe how deviations in wind power in-feed are compensated by conventional generation units and how these deviations influence the power injection at each bus:

$$
\Delta \mathbf{P}_{i n j}=\Psi \cdot \Delta \mathbf{P}_{\mathrm{W}},
$$

where $\Delta \mathbf{P}_{i n j} \in \mathbb{R}^{N_{\mathrm{B}}}$ denotes the changes in power injections at all buses and $\Psi \in \mathbb{R}^{N_{B} \times N_{W}}$ is a matrix containing all GSFs. The amount of balancing power provided by each generator can be arbitrarily defined, e.g. based on the outcome of a reserve procurement process. Here, it is assumed that the change in power output is distributed among all generators in proportion to their nominal maximum output capacity.

To calculate the change in the power injections at each bus, we add the compensation from all generators connected to each bus and subtract the wind power deviation at the bus where the wind power plant is connected. If the wind power plant $w$ is connected to bus $m$, GSF for this node is calculated as:

$$
\Psi_{(m, w)}=\frac{\sum_{n \in \text { node } m} P_{G(n)}^{\max }}{\sum_{j=1}^{\mathrm{N}_{G}} P_{G(j)}^{\max }}-1,
$$

where $P_{G(n)}^{\max }$ denotes the maximum generation capacity of a conventional generation unit $n$. The entries of the other nodes $i$ are given by:

$$
\Psi_{(i, w)}=\frac{\sum_{n \in \text { node } i} P_{G(n)}^{\max }}{\sum_{j=1}^{\mathrm{N}_{\mathrm{G}}} P_{G(j)}^{\max }} .
$$

\section{Line Flow Sensitivity Factors}

When wind power injections deviate from the initial forecast value, the power flows in the system vary accordingly. The actual line flow on branch $i-j$ can be regarded as the sum of the line flow at the forecasted operating point $P_{i j}^{0}$ and a deviation $\Delta P_{i j}$ :

$$
P_{i j}=P_{i j}^{0}+\Delta P_{i j} .
$$

$P_{i j}^{0}$ is calculated using the full $\mathrm{AC}$ power flow equations and can be referred to as the forecasted line flow. To assess the effect of wind power fluctuations on the line flows, we investigate the line flow deviations $\Delta \mathbf{P}_{\ell} \in \mathbb{R}^{N_{\ell}}$ as a function of the wind in-feed deviations $\Delta \mathbf{P}_{\mathrm{W}}$ :

$$
\Delta \mathbf{P}_{\ell}=f\left(\Delta \mathbf{P}_{\mathrm{W}}\right) .
$$

In full $\mathrm{AC}$ power flow equations, the relationship between wind in-feeds and line flow deviations is not linear. However, given that wind power deviations will be small compared to the overall generation in the system, we assume that the line flow changes can be sufficiently well described through a linear relationship. In the following, we illustrate how to approximate (6) as a linear function using Generalized Generation Distribution Factors (GGDFs) [9]:

$$
\Delta \mathbf{P}_{\ell}=\boldsymbol{\Gamma} \cdot \Delta \mathbf{P}_{\mathrm{W}},
$$

where $\Gamma \in \mathbb{R}^{\mathrm{N}_{\ell} \times \mathrm{N}_{\mathrm{W}}}$ is a matrix composed of GGDFs, mapping wind in-feed deviations $\Delta \mathbf{P}_{\mathrm{W}}$ to line flow changes $\Delta \mathbf{P}_{\ell}$. This linearisation will allow us to identify appropriate security margins for transmission lines.

We now present the necessary steps to derive GGDFs based on both $\mathrm{DC}$ and $\mathrm{AC}$ power flow. Considering that sensitivity factors based on $\mathrm{AC}$ power flow not only enable the investigation of active power flow but also yield results on reactive and apparent power flow changes on transmission lines, calculation of sensitivity factors for apparent power flow is also discussed.

1) Generalized Generation Distribution Factors (GGDF): GGDFs describe how line flow changes react to wind power deviations in a linearised way, and can be calculated according to

$$
\begin{aligned}
\Delta \mathbf{P}_{\ell} & =\frac{\partial \mathbf{P}_{\ell}}{\partial \mathbf{P}_{i n j}} \cdot \frac{\partial \mathbf{P}_{i n j}}{\partial \mathbf{P}_{\mathrm{W}}} \cdot \Delta \mathbf{P}_{\mathrm{W}}=\boldsymbol{\Lambda} \cdot \boldsymbol{\Psi} \cdot \Delta \mathbf{P}_{\mathrm{W}} \\
& =\boldsymbol{\Gamma} \cdot \Delta \mathbf{P}_{\mathrm{W}} .
\end{aligned}
$$

Here, $\boldsymbol{\Psi}$ contains the GSFs which are calculated as in (3) and (4). The matrix $\boldsymbol{\Lambda} \in \mathbb{R}^{\mathrm{N}_{\ell} \times \mathrm{N}_{\mathrm{B}}}$ consists of the Power Transfer Distribution Factors (PTDFs), which represent the sensitivity of line flow on a given line $i-j$ with regard to a shift of power from bus $m$ to $n$. It can be defined as [10]:

$$
\boldsymbol{\Lambda}_{(i j, m n)}=\frac{\Delta P_{i j}}{\Delta P_{m \rightarrow n}} .
$$

PTDFs can be derived for either DC or AC power flow, and by inserting AC- or DC-PTDFs, we can obtain the corresponding AC- and DC-GGDFs. We now show how to obtain the PTDFs with $\mathrm{DC}\left(\boldsymbol{\Lambda}^{\mathrm{DC}}\right)$ and $\mathrm{AC}\left(\boldsymbol{\Lambda}^{\mathrm{AC}}\right)$ power flow, as well as an $\mathrm{AC}$ sensitivity factor for apparent power flow (AC-STDF, $\Lambda^{\mathrm{S}}$ ).

2) DC Power Transfer Distribution Factor (DC-PTDF): Based on DC power flow formulations, DC-PTDF for line $i-j$ due to a generation shift between bus $m$ and $n$ can be calculated in the following way [11]:

$$
\Lambda_{(i j, m n)}^{\mathrm{DC}}=\frac{\mathbf{X}_{(i, m)}-\mathbf{X}_{(j, m)}-\mathbf{X}_{(i, n)}+\mathbf{X}_{(j, n)}}{x_{i j}},
$$

where $x_{i j}$ denotes the reactance of the transmission line $i-j$ and $\mathbf{X}_{(i, m)}$ is the entry in $i$ th row and the $m$ th column of the bus reactance matrix $\mathbf{X}$.

3) AC Power Transfer Distribution Factor (AC-PTDF): AC-PTDFs are obtained by linearising the AC power flow around the forecasted operating point and are thus subject to changes in actual system operating points.

Consider first the sensitivity of system states (i.e. voltage 
magnitudes and angles at each bus) with regard to changes in power injections [12]:

$$
\left[\begin{array}{l}
\Delta \boldsymbol{\Theta} \\
\Delta \mathbf{V}
\end{array}\right]=\mathbf{J}_{p x}^{-1}\left[\begin{array}{l}
\Delta \mathbf{P}_{i n j} \\
\Delta \mathbf{Q}_{i n j}
\end{array}\right],
$$

where $\Delta \Theta$ and $\Delta \mathbf{V}$ refer to changes in voltage angles and in voltage magnitudes respectively. $\Delta \mathbf{P}_{i n j}$ and $\Delta \mathbf{Q}_{i n j}$ indicate changes in the active and reactive power injections at each bus, where $\Delta \mathbf{Q}_{i n j}$ is assumed to be zero in this case. $\mathbf{J}_{p x}$ is the Jacobian matrix of power injections and its inverse, $\mathbf{J}_{p x}^{-1}$, is the sensitivity matrix. $\mathbf{J}_{p x}$ consists of the following elements:

$$
\mathbf{J}_{p x}=\left[\begin{array}{ll}
\frac{\partial \mathbf{P}_{i n j}}{\partial \mathbf{V}} & \frac{\partial \mathbf{P}_{i n j}}{\partial \boldsymbol{\Theta}} \\
\frac{\partial \mathbf{Q}_{i n j}}{\partial \mathbf{V}} & \frac{\partial \mathbf{Q}_{i n j}}{\partial \boldsymbol{\Theta}}
\end{array}\right] .
$$

Note that since the voltage magnitude and angle at the slack bus as well as the voltage magnitudes at the PV buses are fixed, the corresponding rows and columns in $\mathbf{J}_{p x}$ should be omitted. Entries in the Jacobian matrix $\mathbf{J}_{p x}$ in (12) can be derived from the power flow equations [13].

Now consider the sensitivity of line flows with regard to changes in system states:

$$
\left[\begin{array}{l}
\Delta \mathbf{P}_{\ell} \\
\Delta \mathbf{Q}_{\ell}
\end{array}\right]=\mathbf{J}_{f x}\left[\begin{array}{c}
\Delta \boldsymbol{\Theta} \\
\Delta \mathbf{V}
\end{array}\right]=\mathbf{J}_{f x} \mathbf{J}_{p x}^{-1}\left[\begin{array}{c}
\Delta \mathbf{P}_{i n j} \\
\Delta \mathbf{Q}_{i n j}
\end{array}\right],
$$

where $\mathbf{J}_{f x}$ is the Jacobian matrix of line active power flows and has the following structure:

$$
\mathbf{J}_{f x}=\left[\begin{array}{ll}
\frac{\partial \mathbf{P}_{\ell}}{\partial \mathbf{V}} & \frac{\partial \mathbf{P}_{\ell}}{\partial \boldsymbol{\Theta}} \\
\frac{\partial \mathbf{Q}_{\ell}}{\partial \mathbf{V}} & \frac{\partial \mathbf{Q}_{\ell}}{\partial \boldsymbol{\Theta}}
\end{array}\right] .
$$

The elements in (14) can be calculated using power flow equations [13]. Eq. (13) describes a linear relation between a change in power injections and a change in line flows. Hence, the AC-PTDF matrix is given by

$$
\boldsymbol{\Lambda}^{\mathrm{AC}}=\mathbf{J}_{f x} \mathbf{J}_{p x}^{-1} .
$$

4) AC Apparent Power Distribution Factors (AC-STDF): In addition to estimating changes in active power flow, $A C$ sensitivity factors can also be extended to reflecting changes in reactive and apparent power flows. Similar to the derivation of AC-PTDFs, AC sensitivity factors for apparent power flows (AC-STDF) on transmission lines can be derived as

$$
\Delta \mathbf{S}_{\ell}=\mathbf{J}_{s x}\left[\begin{array}{c}
\Delta \boldsymbol{\Theta} \\
\Delta \mathbf{V}
\end{array}\right]=\mathbf{J}_{s x} \mathbf{J}_{p x}^{-1}\left[\begin{array}{l}
\Delta \mathbf{P}_{i n j} \\
\Delta \mathbf{Q}_{i n j}
\end{array}\right],
$$

and the AC-STDF matrix is obtained by

$$
\Lambda^{\mathrm{S}}=\mathbf{J}_{s x} \mathbf{J}_{p x}^{-1} .
$$

Here, $\mathbf{J}_{p x}$ consists of the same elements as in (12). $\mathbf{J}_{s x}$ is the Jacobian matrix of apparent power flows on branches and has the following structure:

$$
\mathbf{J}_{s x}=\left[\begin{array}{ll}
\frac{\partial \mathbf{S}_{\ell}}{\partial \mathbf{V}} & \frac{\partial \mathbf{S}_{\ell}}{\partial \boldsymbol{\Theta}}
\end{array}\right] .
$$

The elements of $\mathbf{J}_{s x}$ in (18) can be obtained by:

$$
\frac{\partial S_{i j}}{\partial X}=\frac{\partial S_{i j}}{\partial P_{i j}} \cdot \frac{\partial P_{i j}}{\partial X}+\frac{\partial S_{i j}}{\partial Q_{i j}} \cdot \frac{\partial Q_{i j}}{\partial X},
$$

where $X$ can refer to either voltage magnitude $V$ or voltage angle $\theta$. Since we have $S_{i j}=\sqrt{P_{i j}^{2}+Q_{i j}^{2}}$, (19) can be simplified as:

$$
\frac{\partial S_{i j}}{\partial X}=\frac{P_{i j}^{0}}{S_{i j}^{0}} \cdot \frac{\partial P_{i j}}{\partial X}+\frac{Q_{i j}^{0}}{S_{i j}^{0}} \cdot \frac{\partial Q_{i j}}{\partial X} .
$$

Here, $S_{i j}^{0}, P_{i j}^{0}$ and $Q_{i j}^{0}$ are the apparent power flow, active power flow and reactive power flow on line $i-j$ at forecasted operating point and can be regarded as constants.

Substituting active power flow $\mathbf{P}$ with apparent power flow $\mathbf{S}$ in (8), we obtain a similar relationship for apparent power flow on transmission lines:

$$
\Delta \mathbf{S}_{\ell}=\Lambda^{\mathrm{S}} \cdot \mathbf{\Psi} \cdot \Delta \mathbf{P}_{\mathrm{W}} .
$$

\section{Calculation of Uncertainty Margins}

With the liberalization of electricity market and the increasing intensity of cross-border trading, it becomes more challenging to keep the security margins which are necessary to maintain $\mathrm{N}-1$ security. The determination of appropriate security margins is particularly challenging in systems with high shares of renewables, since the fluctuations incurred by forecast errors will cause deviations from the expected system operating point. In order to maintain system security, Transmission System Operators (TSOs) usually incline to overestimate the necessary uncertainty margins. Although higher margins lead to more secure operation, they also result in increasing costs of operation. Therefore, addressing the issue with appropriate calculations instead of making decisions based on intuitive analysis may lower costs and enhance security in the system [2].

The derivation of the line uncertainty margins follows the definitions in [5]. Consider a constraint on the transmission capacity of line $i-j$ in the following form:

$$
P_{i j} \leq P_{i j}^{\max },
$$

where $P_{i j}$ is the actual line flow and $P_{i j}^{\max }$ is the line capacity limit. Introducing the uncertainty of the wind in-feeds, this constraint becomes:

$$
P_{i j}^{0}+\Delta P_{i j} \leq P_{i j}^{\max } .
$$

Assuming that the line flow deviations can be approximated as a linear function of the wind power deviation, we can express the constraint as:

$$
P_{i j}^{0}+\boldsymbol{\Gamma}_{(i j, \cdot)} \cdot \Delta \mathbf{P}_{\mathrm{W}} \leq P_{i j}^{\max },
$$

where $\boldsymbol{\Gamma}_{(i j, \cdot)}$ contains GGDFs for branch $i-j$ with regard to all wind power generators. The first term $P_{i j}^{0}$ represents the load flow at forecasted operating point, while the second term $\boldsymbol{\Gamma}_{(i j, \cdot)} \cdot \Delta \mathbf{P}_{\mathrm{W}}$ represents the estimated line flow deviations. 
Eq. (24) is not tractable due to the random nature of $\Delta \mathbf{P}_{\mathrm{W}}$. Instead, we substitute (24) with a chance constraint:

$$
\mathbb{P}\left\{P_{i j}^{0}+\boldsymbol{\Gamma}_{(i j, \cdot)} \cdot \Delta \mathbf{P}_{\mathrm{W}} \leq P_{i j}^{\max }\right\} \geq 1-\varepsilon,
$$

where $\varepsilon$ is the maximum acceptable violation probability and is chosen by the user. By applying the properties of normal distribution [14], (25) can be rewritten as:

$$
P_{i j}^{0}+\Phi^{-1}(1-\varepsilon) \sqrt{\boldsymbol{\Gamma}_{(i j, \cdot)} \boldsymbol{\Sigma}_{\mathrm{W}} \boldsymbol{\Gamma}_{(i j, \cdot)}^{\mathrm{T}}} \leq P_{i j}^{\max },
$$

which can be rearranged as

$$
P_{i j}^{0} \leq P_{i j}^{\max }-\Phi^{-1}(1-\varepsilon) \sqrt{\boldsymbol{\Gamma}_{(i j, \cdot)} \boldsymbol{\Sigma}_{\mathrm{W}} \boldsymbol{\Gamma}_{(i j, \cdot)}^{\mathrm{T}}} .
$$

$\Phi^{-1}(1-\varepsilon)$ is the inverse cumulative distribution function of standard normal distribution, evaluated at probability $1-\varepsilon$. If we consider the lower bound of transmission capacity,

$$
P_{i j}^{0}+\Delta P_{i j} \geq-P_{i j}{ }^{\max },
$$

we can derive the following result similarly:

$$
P_{i j}^{0}-\Phi^{-1}(1-\varepsilon) \sqrt{\boldsymbol{\Gamma}_{(i j, \cdot)} \boldsymbol{\Sigma}_{\mathrm{W}} \boldsymbol{\Gamma}_{(i j, \cdot)}^{\mathrm{T}}} \geq-P_{i j}^{\max },
$$

which can be rearranged to

$$
P_{i j}^{0} \geq-P_{i j}^{\max }+\Phi^{-1}(1-\varepsilon) \sqrt{\boldsymbol{\Gamma}_{(i j, \cdot)} \boldsymbol{\Sigma}_{\mathrm{W}} \boldsymbol{\Gamma}_{(i j, \cdot)}^{\mathrm{T}}},
$$

Note that the term $\Phi^{-1}(1-\varepsilon) \sqrt{\boldsymbol{\Gamma}_{(i j,)} \boldsymbol{\Sigma}_{\mathrm{W}} \boldsymbol{\Gamma}_{(i j, \cdot)}^{\mathrm{T}}}$ is always positive when $\varepsilon<0.5$. For the constraints (27) and (30), this term represent a tightening of the constraint, meaning a decrease in available capacity. This decrease can be interpreted as the uncertainty margin which is necessary to keep the system secure with a probability $1-\varepsilon$. The uncertainty margin for a given a confidence level of $1-\varepsilon$ is thus defined as follows:

$$
\Omega_{i j}=\Phi^{-1}(1-\varepsilon) \sqrt{\boldsymbol{\Gamma}_{(i j, \cdot)} \boldsymbol{\Sigma}_{\mathrm{W}} \boldsymbol{\Gamma}_{(i j, \cdot)}^{\mathrm{T}}} .
$$

Eq. (31) is valid for calculating the line uncertainty margin for both AC and DC power flow, by inserting the corresponding GGDF (for active or apparent power flow). Note that the left hand side of (26) and (29) represent the estimated upper and lower quantiles of the line flows (evaluated at probability $1-\varepsilon$ ), and that those quantiles are symmetric around the operating point.

\section{CAse Study}

\section{A. Test System}

The aforementioned methods of modelling RES propagations are implemented on the IEEE RTS96 System (Fig. 1). Wind power in-feed is assumed to be connected at Bus 8 and Bus 15, with an installed capacity of $500 \mathrm{MW}$ and 700 MW respectively. The forecast value of wind power output is assumed to be $25 \%$ of the installed capacity, i.e. $125 \mathrm{MW}$ and 175 MW respectively. In the base case scenario, the standard deviation $\sigma_{\mathrm{W}}$ for each wind power plant is $7.5 \%$ of the total installed capacity. We assume that the forecast errors are independent, such that $\boldsymbol{\Sigma}_{\mathrm{W}}=\operatorname{diag}\left(\sigma_{\mathrm{W}}^{2}\right)$.

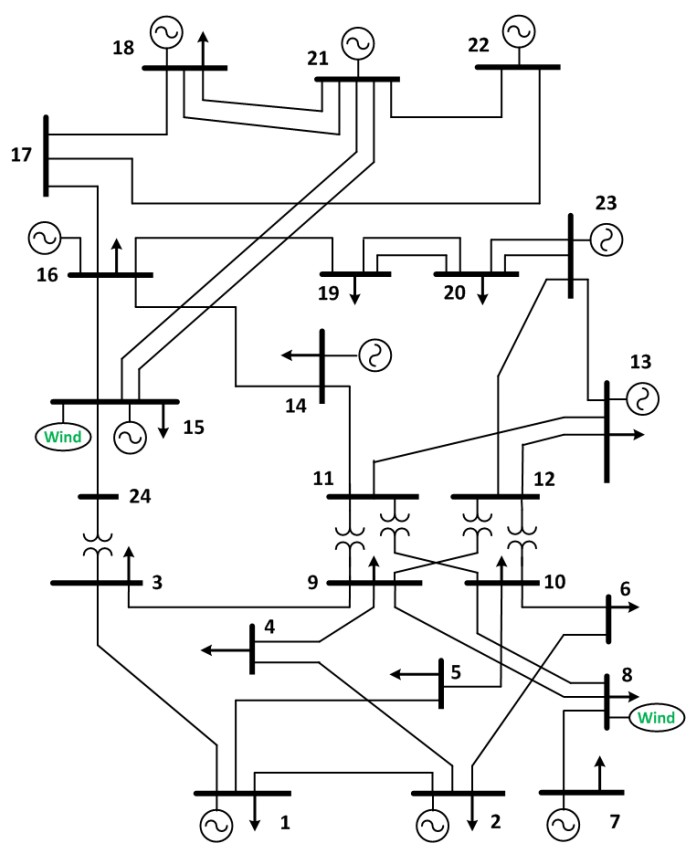

Fig. 1. IEEE RTS96 system with wind power in-feed at Bus 8 and Bus 15

\section{B. Line Flow Changes}

For a given change in the wind power injections, the corresponding deviations in line flow from forecast values are calculated using both DC and AC sensitivity factors. The results are compared with the actual changes obtained by performing full AC power flow calculation. Root-MeanSquare Errors (RMSE) are calculated to get an overview of approximation accuracy.

Fig. 2 shows the estimated line flow deviations using DCand AC-GGDFs in comparison to actual line flow change, assuming $\Delta P_{W}=20 \mathrm{MW}$ at Bus 15 . There is only small discrepancy between estimated line flow changes and actual line flow change in both $\mathrm{AC}$ and $\mathrm{DC}$ scenarios. In this particular case, RMSEs of AC and DC linear approximations are $0.0532 \mathrm{MW}$ and $0.0948 \mathrm{MW}$. Considering that line flow changes have an order of magnitude of $1 \mathrm{MW}$, both errors are acceptable, whereas AC sensitivity factor yields relatively more accurate estimation.

\section{Uncertainty Margins}

Given an acceptable violation probability $\varepsilon$ and the covariance matrix of wind power forecast errors $\boldsymbol{\Sigma}_{\mathrm{W}}$, the uncertainty margins can be calculated by applying DC- and AC-GGDFs in (31). As explained in Section III, the uncertainty margins represent an estimate of the quantiles of the line flow change, evaluated at probability $1-\varepsilon$. One possibility to assess the accuracy of linearised methods is thus to compare the estimated quantiles with the empirical quantiles obtained for full AC power flow in an Monte Carlo simulation (MCS). The margins are calculated with $\varepsilon=0.05$. The results are compared to the empirical margins (the 5\% and $95 \%$ empirical quantiles) obtained by MCS with full AC power flow, where 
Active Power Flow Changes on Transmission Lines

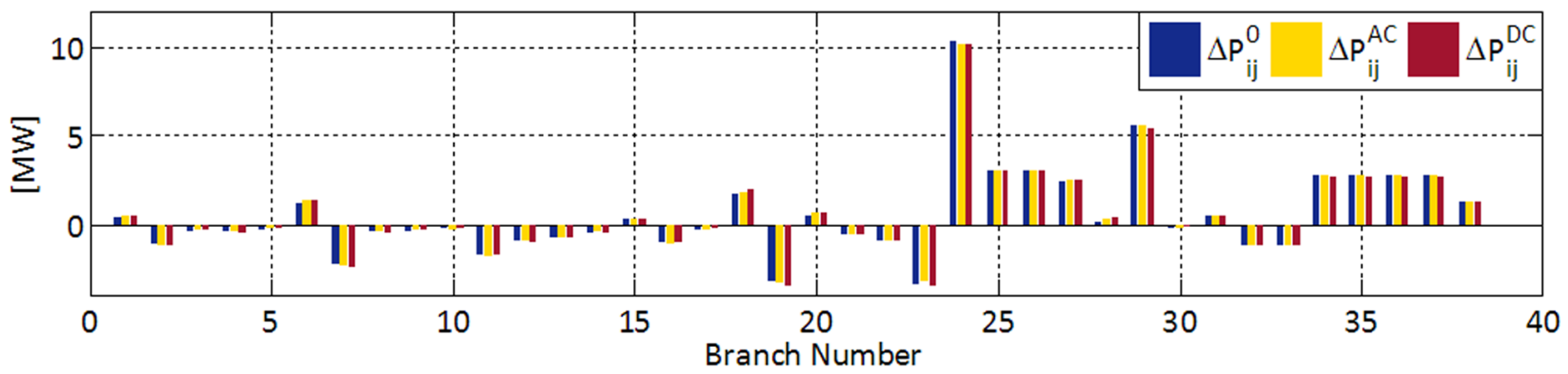

Fig. 2. Comparison of the line flow changes calculated with the full AC equations $\left(\Delta P_{i j}^{0}\right)$, and the line flow changes estimated using AC-GGDFs $\left(\Delta P_{i j}^{A C}\right)$ and DC-GGDFs $\left(\Delta P_{i j}^{D C}\right)$.
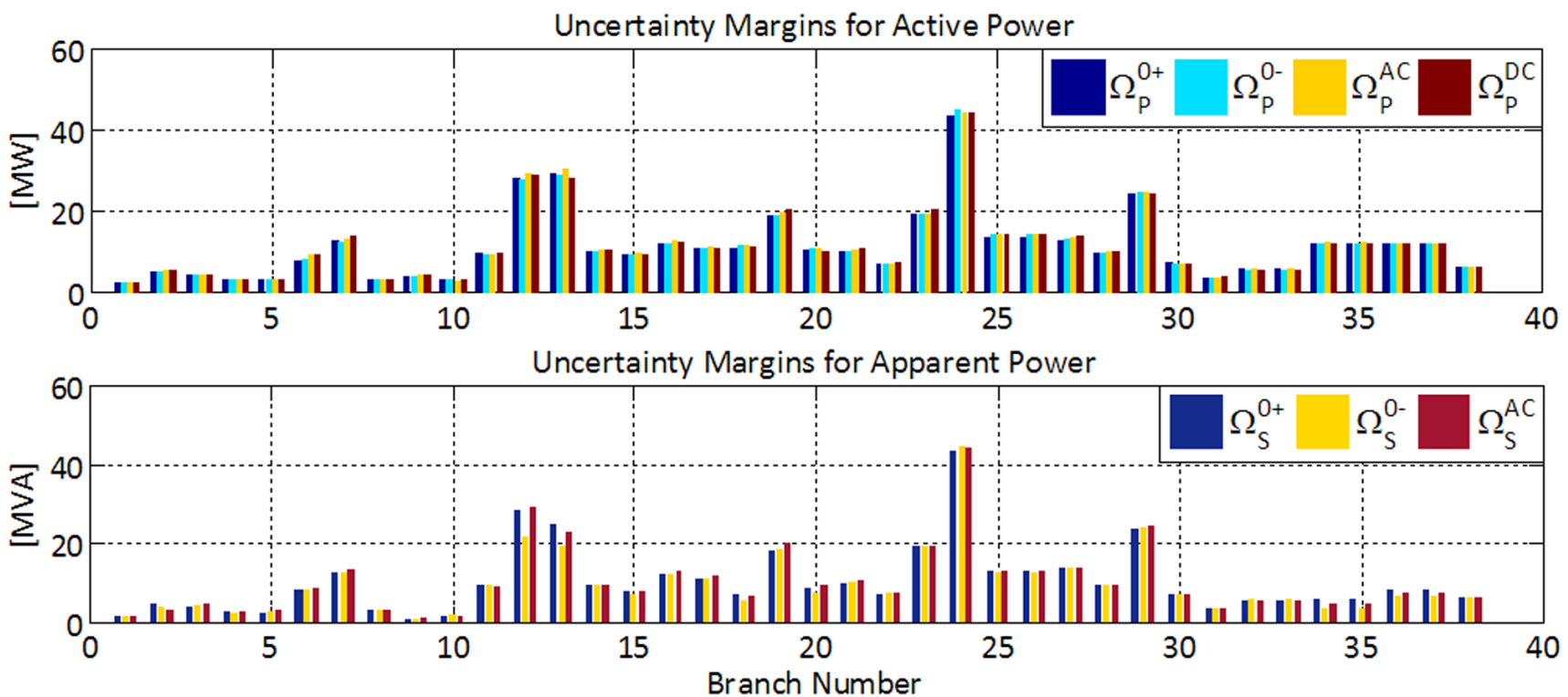

Fig. 3. Upper part: Uncertainty margins for active power flow. Comparison of the empirical uncertainty margins obtained by MCS ( $\Omega_{\mathrm{P}}^{0+}$ for upper quantile and $\Omega_{\mathrm{P}}^{0-}$ for lower quantile), with the uncertainty margins estimated by the AC and DC linearisation, $\left(\Omega_{\mathrm{P}}^{\mathrm{AC}}\right.$ and $\left.\Omega_{\mathrm{P}}^{\mathrm{DC}}\right)$. Lower part: Uncertainty margins for apparent power flow. Comparison of the empirical uncertainty margins obtained by MCS $\left(\Omega_{\mathrm{S}}^{0+}\right.$ for upper quantile and $\Omega_{\mathrm{S}}^{0-}$ for lower quantile), with the uncertainty margins estimated by the AC linearisation $\Omega_{\mathrm{S}}^{\mathrm{AC}}$.

the number of samples is 10,000 . Note that in the full AC equations, the relation between the wind power deviation and the line flow change is non-linear. This means that although the wind power deviations are symmetric, the quantiles of the line flows are not necessarily symmetric.

The upper part of Fig. 3 shows the results for active power flow. The empirical uncertainty margins obtained by MCS are denoted by $\Omega_{\mathrm{P}}^{0+}$ (upper quantile) and $\Omega_{\mathrm{P}}^{0-}$ (lower quantile), and the estimated uncertainty margins obtained by the AC and DC linearisation are denoted by $\Omega_{\mathrm{P}}^{\mathrm{AC}}$ and $\Omega_{\mathrm{P}}^{\mathrm{DC}}$. The lower part of Fig. 3 shows the results for apparent power flow. The empirical uncertainty margins obtained by MCS are denoted by $\Omega_{\mathrm{S}}^{0+}$ (upper quantile) and $\Omega_{\mathrm{S}}^{0-}$ (lower quantile), while the estimated uncertainty margin based on the AC linearisation is denoted by $\Omega_{\mathrm{S}}^{\mathrm{AC}}$. The difference between the upper and lower margins is small, indicating that the line flow change is well approximated through a linear relation. The most asymmetry of empirical margins occurs on lines which have large uncertainty margins (e.g. Lines $24,29,19,12,13$ ). This is as expected, since larger line flow deviations imply that the linearisation might be less accurate.

Both AC and DC approximations provide reasonable results on uncertainty calculations. The largest absolute error of active power uncertainty margin (with respect to the mean value of the upper and lower empirical MCS margin) is around 1.5 MW, whereas the constraint tightening of the corresponding lines has a magnitude of over $20 \mathrm{MW}$. Over $85 \%$ of the absolute errors of apparent power margins are within 1 MVA. RMSEs of AC and DC estimations are found to be 0.5036 MW and 0.5963 MW, respectively. The exact number may vary according to simulation, but it gives an overall indication that the AC sensitivity factors model provides results that are 


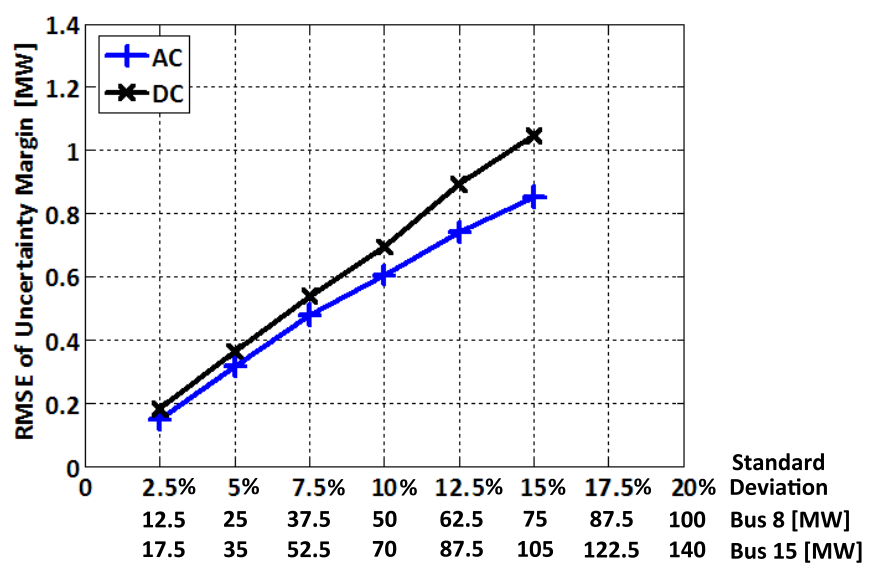

Fig. 4. RMSE of Uncertainty Margins Based on AC and DC Linear Approximation Models with Varying Standard Deviation Level

comparatively closer to Monte Carlo Simulation. Furthermore, the RMSE of AC apparent power uncertainty margin is 0.9004 MVA, which is also acceptable.

\section{Sensitivity Analysis Regarding Forecast Uncertainty}

Since wind power in-feed fluctuations are modelled as random variables, the forecast accuracy represented by covariance matrix impacts the solution of the optimization problem. Larger magnitude of $\sigma_{\mathrm{W}}$ implies that there is a higher probability that the system may be further away from initial forecasted operating point. In this analysis, the $\sigma_{\mathrm{W}}$ of both wind in-feed aggregations at Bus 8 and Bus 15 are increased stepwise from $2.5 \%$ to $15 \%$ of their installed capacity. RMSE of the uncertainty margins by both $\mathrm{AC}$ and DC linear approximation methods is calculated for each scenario.

As can be seen from Fig. 4, RMSEs of both AC and DC approximation models increase almost linearly with standard deviation level. This is because an increase in standard deviation results in larger deviations from the initially forecasted operating point. Hence the linearising assumptions may not be valid any more. Furthermore, as the standard deviation increases, the relative prediction accuracy of the AC model as compared to the DC model is increasing. In the scenario where the standard deviation level reaches $15 \%$, the RMSE of uncertainty margin yielded by $\mathrm{AC}$ sensitivity factor is approximately 0.2 MW lower than the DC model. Therefore, for situations where the wind power forecast reliability level is low, AC model is more suitable for the uncertainty margin estimation.

\section{CONCLUSION}

In this paper, line flow changes due to wind in-feed fluctuations are modelled using linearisation methods. Two models based on DC and linearised AC power flow are introduced and compared. Sensitivity factors are defined and used to estimate both line flow variations due to wind in-feed fluctuations and appropriate uncertainty margins to secure the system against those deviations are derived.
The modelling methods are implemented on a test system with wind power in-feed. Results for the estimation of line flow changes using the AC and DC methods are compared with the actual line flow changes computed with the full AC power flow equations. Further, the uncertainty margins obtained by the AC and DC methods are compared with the empirical quantiles obtained with an Monte Carlo Simulation based on the full AC power flow. Finally, a sensitivity analysis with respect to the forecast accuracy is conducted.

The simulation results show that both the AC and DC models provide reasonable results. The $\mathrm{AC}$ model has an overall better performance than the DC model and can also calculate uncertainty margins for apparent power flow, which has a more direct link to line currents. In future work, we plan to extend the $\mathrm{AC}$ uncertainty margins to voltage and current constraints, and to incorporate the uncertainty margins in both Optimal Power Flow and contingency analysis problems.

\section{ACKNOWLEDGMENT}

This research work has been carried out within the scope of the project "Innovative tools for future coordinated and stable operation of the pan-European electricity transmission system (UMBRELLA)", supported under the 7th Framework Programme of the European Union, grant agreement 282775.

\section{REFERENCES}

[1] World Wind Energy Association, "Key Statistics of World Energy Report 2013," http://www.wwindea.org/webimages/WWEA WorldWindReportKeyFigures_2013.pdf (Date of access: June 8, 2014).

[2] H. Zhang and P. Li, "Probabilistic analysis for optimal power flow under uncertainty," Generation, Transmission Distribution, IET, vol. 4, no. 5 , pp. 553-561, May 2010.

[3] M. Vrakopoulou, K. Margellos, J. Lygeros, and G. Andersson, "Probabilistic guarantees for the N-1 security of systems with wind power generation," in PMAPS 2012, Istanbul, Turkey, 2012.

[4] D. Bienstock, M. Chertkov, and S. Harnett, "Chance-constrained optimal power flow: Risk-aware network control under uncertainty," SIAM Review, vol. 56, no. 3, pp. 461-495, 2014.

[5] L. Roald, F. Oldewurtel, T. Krause, and G. Andersson, "Analytical reformulation of security constrained optimal power flow with probabilistic constraints," in PowerTech, 2013 IEEE Grenoble, June 2013, pp. 1-6.

[6] M. Vrakopoulou, M. Katsampani, K. Margellos, J. Lygeros, and G. Andersson, "Probabilistic security-constrained ac optimal power flow," in Powertech, 2013 IEEE Grenoble, June 2013, pp. 1-6.

[7] A. Kumar and S. Srivastava, "Ac power transfer distribution factors for allocating power transactions in a deregulated market," IEEE Power Engineering Review, vol. 22, no. 7, pp. 42-43, July 2002.

[8] North American Electric Reliability Corporation, "Introduction to IDC Factors," http://www.nerc.com/comm/oc/idc\%20training\%20dl/idc\%20factors.pdf (Date of access: September 21, 2014).

[9] W. Y. Ng, "Generalized generation distribution factors for power system security evaluations," IEEE Trans. Power App. Syst., vol. PAS-100, no. 3, pp. 1001-1005, March 1981.

[10] A. Wood, B. Wollenberg, and G. Sheblé, Power Generation, Operation and Control, 3rd Edition. John Wiley \& Sons, 2014.

[11] R. Christie, B. Wollenberg, and I. Wangensteen, "Transmission management in the deregulated environment," Proceedings of the IEEE, vol. 88, no. 2, pp. 170-195, Feb 2000.

[12] A. Kumar, S. Srivastava, and S. Singh, "Available transfer capability (atc) determination in a competitive electricity market using ac distribution factors," Electric Power Components and Systems, vol. 32, no. 9, pp. 927-939, 2004.

[13] A. Bergen and V. Vittal, Power Systems Analysis. Prentice Hall, 2000.

[14] S. Boyd and L. Vandenberghe, Convex Optimization. Cambridge University Press, 2004. 